\title{
THERMAL PULSES AND THE FORMATION OF PLANETARY NEBULA SHELLS
}

\author{
Alvio Renzini \\ Dipartimento di Astronomia, Università di Bologna, Bologna, Italy
}

\section{INTRODUCTION}

Over the past decade a comprehensive, semiquantitative theoretical scenario for the final evolutionary stages of low and intermediate mass stars has been progressively elaborated and refined. It concerns the envelope ejection terminating the Asymptotic Giant Branch (AGB) phase, the AGB to Planetary Nebula (PN) transition, the fading and possible rejuvenation of $\mathrm{PN}$ nuclei, the formation processes of hydrogen-deficient stars, and the final production of white dwarfs (WD) of the DA and non-DA varieties (Renzini 1979, 1981a, 1981b, 1982, 1983, Iben \& Renzini 1983 , Iben et al. 1983, Iben 1984, 1985, 1987, Iben \& Tutukov 1984, Iben \& MacDonald 1985, 1986). In developing this scenario several important results of stellar evolution and hydrodynamical calculations have been incorporated, including in particular those of Paczyǹski (1971), Wood (1974), Härm \& Schwarzschild (1975), Schönberner $(1979,1983)$, and Tuchman, Sack \& Barkat (1979).

In the present communication an attempt is presented to further enrich this scenario, by explicitly considering some aspects which heretofore have remained insufficiently explored. These include the relation between thermal pulses and the envelope ejection (EE) at the tip of the AGB, with emphasis on the diversities that this relation may present depending on the initial mass of evolving stars (Section 2). The implications of EE details for the subsequent, Post-AGB evolution are then cursorily explored for the various cases. In Section 3 the question of the AGB to PN transition time is revisited, and the consequences of the use of a misleading definition of the nebular age are briefly discussed. Finally, in Section 4 some concluding considerations of general validity are recalled, together with a few elements which may be useful for the definition of the most urgent observational investigations. 


\section{THERMAL PULSES AND ENVELOPE EJECTION}

The EE at the tip of the AGB is currently ascribed to a short phase of intense mass loss, conventionally called superwind to distinguish it from the regular red giant wind. This follows from the typical empirical values of the mass, radius and expansion velocity of $\mathrm{PN}$ shells, which require mass loss rates of $\sim 10^{-5}$ $10^{-4} M_{\odot} \mathrm{yr}^{-1}$, having operated for $\sim 10^{3}-10^{4} \mathrm{yr}$. This, and nothing more, is what we mean by superwind, and the identification of the superwind phase with the $\mathrm{OH} / \mathrm{IR}$ phase then follows very naturally (for oxygen rich stars, cf. Habing, this volume). A superwind phase can also be identified for carbon stars, i.e. with highly obscured, carbon rich objects (cf. Knapp, this volume). After the superwind has removed most of the hydrogen-rich envelope the star leaves the AGB, initiates its migration towards high temperatures, and the superwind gets quenched leaving a residual envelope mass $M_{\mathrm{e}}^{\mathrm{R}}$. To avoid semantic confusions it is worth recalling that two other kinds of stellar wind respectively precede and follow the superwind phase: the so-called regular red giant wind operating during the AGB phase, with a typical rate some 100 times smaller than the superwind, and the subsequent fast wind emitted during the PN stage.

Thermal pulses, also known as helium shell flashes, have often been regarded as a possible trigger for the EE (e.g. Rose \& Smith 1972, Trimble \& Sackman 1978, Tuchman \& Barkat 1980). Indeed, following a thermal pulse the surface luminosity increses by $\sim 3 / 4$ of a magnitude (cf. Iben 1982), and the transition from the regular wind to the superwind obviously becomes more likely. It is well known that $\mathrm{PNe}$ and WDs are produced by stars in a rather wide range of initial masses, from $\sim 0.85 M_{\odot}$ up to perhaps $\sim 8 M_{\odot}$, and correspondingly we should expect that the EE may assume different quantitative and qualitative characteristics, depending on the initial mass $M_{\mathrm{i}}$, and the related mass of the hydrogen exhausted core $M_{\mathrm{H}}$. For example, the duration $\Delta t_{\text {peak }}$ of this post-flash luminosity peak is a strong function of $M_{\mathrm{H}}$, with a rough analytic fit giving:

$$
\Delta t_{\text {peak }} \simeq 60 M_{\mathrm{H}}^{-8} \mathrm{yr} .
$$

The variation of the peak duration with $M_{\mathrm{H}}$, coupled with the large variation of the available envelope mass, gives rise to a variety of situations that are explored next.

\subsection{Case A: a low mass, Pupolation II star}

Evolving Population II stars, such as those in galactic globular clusters, have an initial mass $M_{\mathrm{i}} \simeq 0.85 M_{\odot}$. Their mass is then decreased by stellar winds during both the first red giant branch, and the early portion of the AGB. By the time they experience the first thermal pulse on the AGB their core mass has grown to $M_{\mathrm{H}} \simeq 0.54 M_{\odot}$, and their envelope mass $M_{\mathrm{e}}$ has thinned to just a few $10^{-2} M_{\odot}$. With $M_{\mathrm{H}}=0.54 M_{\odot}$, Eq. (1) gives $\Delta t_{\text {peak }} \simeq 10^{4} \mathrm{yr}$, and with a superwind mass loss rate $\dot{M}_{\mathrm{SW}} \simeq 10^{-5} M_{\odot} \mathrm{yr}^{-1}$ the mass that could be lost during one pulse peak 
$\left(\Delta M=\Delta t_{\text {peak }} \dot{M}_{\mathrm{SW}} \simeq 0.1 M_{\odot}\right)$ largely exceeds the envelope mass $M_{\mathrm{e}}$. This implies that in such low mass stars the envelope ejection may be possible during just one thermal pulse, and most likely during the very first one (Renzini \& Fusi Pecci 1988).

The superwind envelope removal during one pulse peak has several important implications for the subsequent evolution of Population II stars. In fact, helium burning Post-AGB stars are produced, and since their luminosity is independent of $M_{\mathrm{e}}$, the fast wind (typical of PN nuclei) is likely to complete the removal of the residual envelope during the bright Post-AGB phase. With $M_{\mathrm{e}}^{\mathrm{R}} \simeq 10^{-3} M_{\odot}$, and a Post-AGB fading time $\sim$ several $10^{5} \mathrm{yr}$, a mass loss rate $\lesssim 10^{-8} M_{\odot} \mathrm{yr}^{-1}$ is indeed sufficient to expose the helium/carbon intershell material, thus producing a hydrogen deficient Post-AGB star which will eventually evolve into a non-DA WD.

This scenario may offer a chance to explain the otherwise puzzling high carbon abundance observed in three out of the four known PNe in the galactic Halo (TorresPeimbert 1984, Clegg 1985), one of which is K648, the PN in the globular cluster M15. With $\mathrm{C} / \mathrm{O} \simeq 10$, these $\mathrm{PNe}$ should have been ejected by AGB carbon stars, which neither are found in galactic globular clusters, nor are produced by current AGB models for $M_{\mathrm{H}} \lesssim 0.6 M_{\odot}$ (cf. Iben \& Renzini 1984). There is therefore both an empirical and a theoretical embarrassment for dredge-up in the precursor having caused the carbon overabundance. In another alternative, the carbon rich wind $\left(X_{C}^{\text {wind }} \simeq 0.2\right)$ from a bare PN nucleous could inplant carbon/helium rich, high density pockets into these very young PNe. In the prototype case of K648 the only photospheric spectral feature is a strong CIV asymmetric absorption (Adams et al. 1984), perhaps suggestive of a $\mathrm{He} / \mathrm{C}$ atmosphere. With $M_{\mathrm{PN}} \simeq 0.01 M_{\odot}$ and an estimated nebular abundance $X_{C}^{\mathrm{PN}} \simeq 0.005$, the $\mathrm{PN}$ would contain $\sim 5 \times 10^{-5} M_{\odot}$ of carbon: too much for having been provided within the K648 lifetime $(\sim 2000$ yr) by the present carbon mass loss rate $X_{\mathrm{C}}^{\text {wind }} \times \dot{M} \simeq 10^{-12} M_{\odot} \mathrm{yr}^{-1}$ estimated by Adams et al. On the other hand, in the carbon inplantation scenario, the nebular carbon lines originate from shocked (and then cooled) high density wind material, rather than from a homogeneous nebula. A much smaller carbon mass may therefore be sufficient to account for the observed emission. In any case, carbon rich $\mathrm{PNe}$ of Population II would have carbon rich nuclei (e.g. Wolf-Rayet type of the WC variety), and carbon rich inhomogeneities, and both predictions are easily testable by Space Telescope (HST) observations.

HST observations of globular clusters will provide further opportunity for a decisive test of this scenario. Indeed, only non-DA WDs should be produced and detected, if in low mass stars the envelope ejection is always linked to a thermal pulse. Taking advantage of the excellent observational conditions at the $\mathrm{CFH}$ telescope, Richer \& Fahlman (1987) may have recently anticipated the space detection of globular cluster WDs, and Ortolani \& Rosino (1987) may have achieved the same result for the cluster $\omega$ Cen. From the location in the $U-(U-V)$ diagram of their six WD candidates in M71, Richer \& Fahlman conclude that most likely they all belong to the non-DA variety, thus providing circumstantial support to the idea of a flash-triggered EE in globular cluster stars. 


\subsection{Case B: a typical old disk star}

As we consider stars of larger and larger initial mass, the core mass $M_{\mathrm{H}}$ at the first thermal pulse tends to increase (although slowly), $\Delta t_{\text {peak }}$ decreases according to Eq. (1), while the available envelope mass obviously increases. The fraction of the envelope $\left(\Delta t_{\text {peak }} \dot{M}_{\mathrm{SW}} / M_{\mathrm{e}}\right)$ that can be lost during one post-flash peak therefore decreases, provided $\dot{M}_{\mathrm{SW}}$ does not increase too rapidly. For a typical disk star, such as the precursors of most $\mathrm{PNe}$ in the solar neighborhood, we can adopt $M_{\mathrm{i}} \lesssim 2 M_{\odot}$, $M_{\mathrm{H}} \simeq 0.65 M_{\odot}, M_{\mathrm{e}} \simeq 1 M_{\odot}$, and $\dot{M}_{\mathrm{SW}} \simeq$ few $10^{-5} M_{\odot} \mathrm{yr}^{-1}$. Correspondingly, we have $\Delta M=\Delta t_{\text {peak }} \dot{M}_{\mathrm{SW}} \simeq$ few $10^{-2} M_{\odot}$, i.e. much less than the available envelope mass $M_{\mathrm{e}}$. Envelope removal during a post-flash peak is therefore relatively infrequent, while the superwind can easily remove most of the envelope during one interpulse period (duration = several $10^{4} \mathrm{yr}$ ) when the star is burning hydrogen in the shell. In this case the production of hydrogen burning Post-AGB stars is then more likely, as so is the formation of DA remnant WDs, in agreement with their larger frequency in the solar neighborhood, compared to non-DAs.

\subsection{Case C: young disk stars}

It is now several years that an embarrassingly large discrepancy persists between theoretical AGB models on one side, and the observations of AGB stars in the Magellanic Clouds on the other side (e.g. Iben \& Truran 1978, Renzini \& Voli 1981, Blanco et al. 1980, Reid \& Mould 1985, and references therein). Theory indeed predicts much more bright AGB stars $\left(M_{\text {bol }} \lesssim-6.0\right)$ than actually observed, and several possible explanations have been suggested. The latest, perhaps most promising of these suggestions moves from the finding of severe convergence problems in thermally pulsing AGB models brighter than this limit, or, equivalently, with core mass $M_{\mathrm{H}} \gtrsim 0.85 M_{\odot}$ (Wood \& Faulkner 1986, Mazzitelli 1987). Actually, shortly after a thermal pulse, the local luminosity may reach dangerously close to the Eddington limit, as the energy released by the flash leaks out through the base of the envelope. It is then speculated that a radiation pressure levitation can lead to a rapid, hydrodynamical ejection of the envelope, as first suggested by Rose \& Smith (1972).

Several aspects of the idea certainly require careful examination. For example, rapid but still quasi-static envelope expansion subtracts energy from the local energy flow, thus potentially providing a self-regulating mechanism contrasting the approach of the local luminosity to the Eddington limit. The use of envelope models neglecting the so-called gravitational energy coefficient $\epsilon_{\mathrm{g}}$ (as done by Wood \& Faulkner) could therefore give misleading indications, especially in this particular case. Certainly, if one wants to study the onset of this radiation pressure instability, rather than dropping the $\epsilon_{\mathrm{g}}$ term, it would actually be more appropriate to include the acceleration term in the equation of hydrostatic equilibrium, thus turning it into the equation of motion! This could be most efficiently obtained by using an implicit hydrodynamics stellar structure program, such as the KEPLER code of Weaver et al. (1978), which is able of generating a sequence of hydrostatic 
evolutionary models, as well as of following the onset and development of an hydrodynamical instability, if any. On the other hand, apart from the limitations of the current approach to the problem, the mere fact that models corresponding to the limits of the observed AGB present numerical and physical difficulties is highly suggestive, as it may offer an attractive solution to a long lasting discrepancy between theory and observations.

Before proceeding further, it is worth emphasizing the physical difference between the pulse triggered envelope ejection discussed in the previous two sections, and this one, based on the radiation pressure mechanism. In the former ones the superwind may be due to a pulsational instability of the whole envelope, like in the case of the transition from the overtone to the fundamental mode, as proposed by Wood (1974). Here, the hydrodynamical runaway seems to develop in a very restricted region, at the base of the hydrogen rich envelope. The large inflation of this region (perhaps followed by recollapse, bounce, and shock formation) would then lead to the rapid ejection of the envelope. The details of the phenomenon remain however totally unexplored, including in particular its timescale and the number of pulses required to complete the envelope removal, which may considerably increase with increasing initial mass.

Observations may greatly help the development of a satisfactory theory for this kind of ejection, which should be experienced by the more massive $\left(3 \lesssim M_{\mathrm{i}} \lesssim 8 M_{\odot}\right)$ and brighter $\left(-6 \lesssim M_{\text {bol }} \lesssim-7\right)$ AGB stars, leaving the more massive Post-AGB remnants $\left(0.85 \lesssim M_{\mathrm{H}} \lesssim 1.06 M_{\odot}\right)$. OH/IR sources in this luminosity range are therefore the natural candidates to look at, although the observational situation is complicated by the relative rarity of such massive AGB stars, compared to lower mass ones. In fact, it could be hard to extract the objects belonging to the real highluminosity tail of the $\mathrm{OH} / \mathrm{IR}$ luminosity distribution, as the apparent tail of the luminosity function may be substantially contaminated by bulk, intrinsically fainter sources having diffused to higher luminosities because of errors, for example in distance modulus. Moreover, the Post-AGB progeny of these massive AGB stars must be searched among the faintest PN nuclei, as the fading time dramatically drops with increasing $M_{\mathrm{H}}$.

On the other hand, these observational difficulties have to be overcome in one way or another, if one wants to understand the final evolutionary stages of $\sim 3$ to $\sim 8 M_{\odot}$ stars, and to assess their contribution to galactic nucleosynthesis. Indeed, with the prompt ejection envisaged in this radiation pressure scenario there is little space for the third dredge-up and envelope burning processes to work, and correspondingly the contribution of $\sim 3$ to $\sim 8 M_{\odot}$ stars to galactic enrichment would be limited to the first and second dredge-ups, with a corresponding drastic reduction over the predictions of Iben \& Truran (1978) and Renzini \& Voli (1981).

Concerning the Post-AGB evolution, Wood \& Faulkner (1986) argue that, thanks to the radiation pressure ejection process, all AGB stars which produce core masses greater than $\sim 0.85 M_{\odot}$ should produce helium rich PN nuclei, and therefore non-DA WD remnants. The fact that Sirius $\mathrm{B}$ is a DA with $M \simeq 1 M_{\odot}$ apparently 
militates against this suggestion, in particular if one rejects the speculative hypothesis of a subsequent hydrogen accretion onto this WD. More likely, for such large $M_{\mathrm{H}}$ values the fading time is too short to allow the complete envelope removal by the fast, Post-AGB wind.

\section{ENVELOPE EJECTION AND POST-AGB TIMESCALES}

\subsection{The AGB to $P N$ Transition}

It is well known that the production of an observable $\mathrm{PN}$ requires a fine tuning between two physically independent timescales: the dispersion time of the nebula, and the transition time $t_{\mathrm{tr}}$ taken by the central star to evolve from the AGB to its hot configuration. For practical purposes $t_{\mathrm{tr}}$ is defined as the time spent by the star to evolve from the superwind quenching somewhere close to the AGB, to the effective temperature $T_{\text {eff }}=30,000 \mathrm{~K}$, high enough for the excitation of the PN. Following this definition, the transition phase begins when the superwind phase ends, and a regular wind resumes. It is also useful to introduce the quantity $M_{\mathrm{e}}^{\mathrm{N}}$, as the envelope mass when the above effective temperature is first reached. Note that in this section one deals with the evolution of the central stars, rather than with the behavior of the ejecta through the protoplanetary phase, an aspect discussed e.g. by Kwok (1987).

In the case of hydrogen burning Post-AGB stars the transition time is primarily controlled by the residual envelope mass $M_{\mathrm{e}}^{\mathrm{R}}$, whatever the physical mechanism responsible for the transition. One can indeed define three relevant timescales, respectively nuclear, wind and thermal:

$$
\begin{array}{cc}
\text { Nuclear } & \tau_{\mathrm{N}}=\frac{X_{\mathrm{e}} \Delta M_{\mathrm{e}}}{L / E_{\mathrm{H}}} \\
\text { Wind } & \tau_{\mathrm{W}}=\frac{\Delta M_{\mathrm{e}}}{\dot{M}} \\
\text { Thermal } & \tau_{\mathrm{th}}=\frac{G M_{\mathrm{H}} M_{\mathrm{e}}^{\mathrm{R}}}{L R},
\end{array}
$$

where $\Delta M_{\mathrm{e}}=M_{\mathrm{e}}^{\mathrm{R}}-M_{\mathrm{e}}^{\mathrm{N}}, L$ is the luminosity during the transition phase, $E_{\mathrm{H}}$ is the energy released by the nuclear burning of one gram of hydrogen, $X_{\mathrm{e}}$ is the envelope hydrogen abundance, $\dot{M}$ is the average wind mass loss rate operating during the transition, and $R$ is the stellar radius at the beginning of the PN phase, conventionally fixed at $T_{\text {eff }}=30,000 \mathrm{~K}$. The first two timescales are simply given by the amount of envelope consumed during the transition, over the rate of this consumption, due to either nuclear burning or mass loss. In the third case what is consumed is the gravitational energy of the envelope, rather than the envelope itself, but the corresponding timescale is defined in a formally equivalent way. The wind timescale depends on the mass loss rate, which is very poorly known for stars in the 
relevant temperature range, when also the regular red giant wind gets quenched, and a the fast wind typical of hot stars is about to start.

Between the first two timescales, nature will automatically choose the shortest for the transition time $t_{\mathrm{tr}}$ :

$$
t_{\mathrm{tr}} \simeq \operatorname{Min}\left(\tau_{\mathrm{N}}, \tau_{\mathrm{W}}\right)
$$

and would actually chose the third $\left(t_{\mathrm{tr}} \simeq \tau_{\mathrm{th}}\right)$ if $M_{\mathrm{e}}^{\mathrm{R}} \lesssim M_{\mathrm{e}}^{\mathrm{N}}$, i.e. when after superwind quenching the envelope is left very far from its thermal balance. It would be astrophysically interesting to assess which one of the various possibilities is practically realized, depending on the stellar initial mass. The existence of so many $\mathrm{PNe}$ with arguably very small nebular age (see next section) indicates that quite often the transition is faster than the nuclear timescale. However, the point worth stressing here is that, whatever the actual timescale, this will in any case depend on the residual envelope mass $M_{\mathrm{e}}^{\mathrm{R}}$, whose value is practically unknown any better than in order of magnitude $\left(\sim 10^{-3} M_{\odot}\right)$. In other terms, nobody knows whether the superwind will cease at, say, $\log T_{\text {eff }}=3.65,3.70,3.75$, or whatever, but these small differences actually imply very large differences in $M_{\mathrm{e}}^{\mathrm{R}}$, and therefore in $t_{\mathrm{tr}}$.

The fact that $t_{\mathrm{tr}}$ remains indeterminate has important implications for our understanding of the Post-AGB evolution. Indeed, as most recently emphasized by Wood \& Faulkner (1986), this implies that Post-AGB timescales remain uncertain by the additive, unknown term $t_{\mathrm{tr}}$, and therefore time marks on evolutionary sequences must be regarded as relative to an arbitrary zero point, even when this is not explicitly emphasized. Moreover, the inavoidably hydrodynamical nature of the superwind envelope ejection strongly supports the notion that sizable fluctuations of $M_{\mathrm{e}}^{\mathrm{R}}$ values are likely even among virtually identical stars, and therefore no strict determinism is possible for the Post-AGB evolution. Pretending the contrary may lead to serious misinterpretations of the observational data.

All the above considerations refer to the case of hydrogen burning Post-AGB stars, i.e. to stars completing the superwind envelope ejection while burning hydrogen during a so called interpulse phase. In the case of helium burning Post-AGB stars, the evolution across the HR diagram is primarily driven by the secular decrease in the luminosity released by the helium burning shell, and is still affected by the residual envelope mass $M_{\mathrm{e}}^{\mathrm{R}}$ (cf. Iben 1984, Wood \& Faulkner 1986). Also in this case the transition time is therefore controlled by $M_{\mathrm{e}}^{\mathrm{R}}$, but the physics is somewhat more complicated, and less straightforward is the relation between $t_{\mathrm{tr}}$ and the basic stellar timescales.

\subsection{What is the Nebular Age?}

The quantity $R_{\mathrm{PN}} / v_{\exp }$ if often used as a measure of the age of optically thin PNe. Here $R_{\mathrm{PN}}$ is the nebular (outer) radius and $v_{\text {exp }}$ is the expansion velocity, which is assumed constant through the nebular evolution. This is a rough definition indeed, good at most for order of magnitude estimates, but which should be used cum grano salis to avoid erroneous conclusions. In fact, the outer edge of a thin nebula in case 
corresponds to the transition from the regular wind to the superwind, and therefore $R_{\mathrm{PN}} / v_{\text {exp }}$ represents the time elapsed since the beginning of the superwind phase, i.e.:

$$
\frac{R_{\mathrm{PN}}}{v_{\exp }} \simeq t_{\mathrm{SW}}+t_{\mathrm{tr}}+t_{\mathrm{PN}}
$$

where $t_{\mathrm{SW}}$ is the duration of the superwind phase $\left(10^{3}-10^{4} \mathrm{yr}\right)$, and $t_{\mathrm{PN}}$ is the proper nebular age, defined as the time elapsed since the first shining of the nebula. As noted by Méndez et al. (1987), $t_{\mathrm{PN}} \simeq R_{\mathrm{PN}} / v_{\text {exp }}$ only if $t_{\mathrm{SW}}$ and $t_{\mathrm{tr}}$ are both negligibly small. This does not appear to be the case in most instances. In principle, one could get rid of the term $t_{\mathrm{Sw}}$, by using the inner, rather than the outer nebular radius, so that:

$$
\frac{R_{\mathrm{PN}}^{\mathrm{inner}}}{v_{\mathrm{exp}}} \simeq t_{\mathrm{tr}}+t_{\mathrm{PN}},
$$

but the indeterminacy of $t_{\mathrm{tr}}$ clearly remains. In this connection, browsing through an atlas of $\mathrm{PNe}$ (e.g. Chu et al. 1987) one can appreciate a number of interesting situations, ranging from butterfly $\mathrm{PNe}$ for which it seems difficult to define either an outer or an inner radius, to filled PNe (such as NGC 6894 or IC 3568) where the inner radius is vanishingly small. Certainly, in these cases the nebular age must be much shorter than $R_{\mathrm{PN}} / v_{\text {exp }}$ (i.e. $t_{\mathrm{PN}} \ll t_{\mathrm{SW}}$ ), while only in an overwhelming minority of cases can $R_{\mathrm{PN}}^{\text {inner }}$ be unambiguously defined.

From the above considerations, it therefore appears that in most istances it is currently impossible to define a precise kinematic age of the nebulae, which unavoidably remains uncertain by at least the first of the two additive terms $t_{\mathrm{SW}}$ and $t_{\mathrm{tr}}$. In conclusion, it appears that both the evolutionary clock and the kinematic clock are affected by zero point uncertainties that cannot be presently eliminated, and their synchronization is correspondingly precluded. It seems therefore fair to firmly discourage the use of diagrams involving an admixture of theoretical evolutionary times and ill-defined nebular ages, such as plots of the luminosity of central stars versus nebular radii. This would in fact be equivalent to use a pair of unsynchronized clocks, each affected by an unknown zero point bias which can even differ from one PN to the next, and from one evolutionary sequence to another. The claimed success of investigations making use of such plots is then more apparent than real, as the occasionally reasonable results (such as for example an average mass $<M_{\mathrm{H}}>\simeq 0.6 M_{\odot}$ for PN nuclei) actually follow from the very strong mass dependence of the fading time of Post-AGB stars, where $t_{\mathrm{f}} \propto \sim M_{\mathrm{H}}{ }^{-8}$ (cf. Iben \& Renzini 1983). For example, this dependence ensures that even a factor of 2 error in the age translates into only a $\sim 10 \%$ error in the inferred mass $M_{\mathrm{H}}$. Moreover, any desired fine adjustement in the inferred value of $\left\langle M_{H}\right\rangle$ is easily achieved by fudging with the arbitrary choice for the zero point of the Post-AGB timescale. 


\section{CONCLUSIONS}

As a conclusion, I would like to summarize here some of the main points so far discussed.

- Stellar evolution theory predicts the production of single star PN nuclei and WDs in the mass range from $\sim 0.54 M_{\odot}$ up to at least $\sim 1.06 M_{\odot}$, which corresponds to the minimum core mass for non-degenerate carbon ignition.

- Thermal pulses are likely to cause envelope ejection either for low mass, Population II stars $\left(M_{\mathrm{i}} \lesssim 1 M_{\odot}\right)$, or for relatively young Population I stars $\left(M_{\mathrm{i}} \gtrsim 3 M_{\odot}\right)$.

- In older disk stars $\left(1 \lesssim M_{\mathrm{i}} \lesssim 3 M_{\odot}\right)$, i.e. in most cases, superwind envelope ejection appears more likely during one interpulse phase, rather than in coincidence with a thermal pulse.

- It appears that progress in understanding the final evolutionary stages of the more massive $\mathrm{PN} / \mathrm{WD}$ producers (i.e. stars with $3 \leqslant M_{\mathrm{i}} \leqslant 8 M_{\odot}$ ) can be achieved by concentrating on the study of the high luminosity tail of the luminosity function of $\mathrm{OH} / \mathrm{IR}$ and IR sources, and on their likely progeny, i.e. the bolometrically faintest PN nuclei.

Acknowledgments. I am grateful to Dr.s Robin Clegg, Rolf Kudritzki, Leon Lucy, and R.H. Méndez for useful and stimulating discussions, and to ESO for its hospitality during the period in which part of the points discussed in this paper have been worked out. This research has been partly supported through grants of the Italian Ministry of Public Education (MPI) for the scientific research.

\section{REFERENCES}

Adams, S., Seaton, M.J., Howarth, I.D., Aurière, M., Walsh, J.R. 1984. M.N.R.A.S. 207: 471

Blanco, V.M., McCarthy, M.F., Blanco, B.M. 1980. Ap. J. 242: 938

Chu, Y.-H., Jacoby, G.H., Arendt, R. 1987. Ap. J. Suppl. 64: 529

Clegg, R.E.S. 1985. Production and Distribution of CNO Elements ed.

I.J. Danziger, F. Matteucci, \& K. Kjär (Garching: ESO), p. 261

Härm, R., Schwarzschild, M. 1975. Ap. J. 200: 324

Iben, I.Jr. 1982. Ap. J. 260: 821

Iben, I.Jr. 1984. Ap. J. 277: 333

Iben, I.Jr. 1985. Quart.J.R.A.S. 26: 1

Iben, I.Jr. 1987. Late Stages of Stellar Evolution, ed. S. Kwok \& S.R. Pottasch (Dordrecht: Reidel), p. 175

Iben, I.Jr., Kaler, J.B., Truran, J.W., Renzini, A. 1983. Ap. J. 277: 333

Iben, I.Jr., MacDonald, J. 1985. Ap. J. 296: 540

Iben, I.Jr., MacDonald, J. 1986. Ap. J. 301: 164

Iben, I.Jr., Renzini, A. 1983. Ann. Rev. Astron. Astrophys. 21: 271 
Iben, I.Jr., Renzini, A. 1984. Physics Reports. 105: 329

Iben, I.Jr., Truran, J.W. 1978. Ap. J. 220: 980

Iben, I.Jr., Tutukov, A.V. 1984. Ap. J. 282: 615

Kwok, S. 1987. Late Stages of Stellar Evolution, ed. S. Kwok \& S.R. Pottasch

(Dordrecht: Reidel), p. 321

Mazzitelli, I. 1987. Mem.S.A.It. 58: 117

Méndez, R.H., Kudritzki, R.P., Herrero, A., Husfeld, D., Groth, H.G. 1987. preprint

Ortolani, S., Rosino, L. 1987. Astron. Astrophys. 185, 102

Paczyǹski, B. 1971. Acta Astronomica. 21: 417

Renzini, A. 1979. Stars and Star Systems: ed. B. Westerlund (Dordrecht: Reidel), p. 155

Renzini, A. 1981a. Physical Processes in Red Giants: ed. I. Iben Jr. \& A. Renzini (Dordrecht: Reidel), p. 431

Renzini, A. 1981b. Mass Loss and Stellar Evolution: ed. C. Chiosi \& R. Stalio (Dordrecht: Reidel), p. 319

Renzini, A. 1982. Wolf-Rayet Stars: ed. C.W.H. de Loore \& A.J. Willis

(Dordrecht: Reidel), p. 413

Renzini, A. 1983a. Planetary Nebulae: ed. D.R. Flower (Dordrecht: Reidel), p. 267

Renzini, A., Fusi Pecci, F. 1988. Ann. Rev. Astron. Astrophys. in press

Renzini, A., Voli, M. 1981. Astron. Astrophys. 94, 175

Rose, W.K., Smith, R.L. 1972. Ap. J. 173: 385

Schönberner, D. 1979. Astron. Astrophys. 79: 108

Schönberner, D. 1983. Ap. J. 272: 708

Torres-Peimbert, S. 1984. Stellar Nucleosynthesis: ed. C. Chiosi \& A. Renzini

(Dordrecht: Reidel), p. 3

Trimble, V., Sackman, I.-J. 1978. M.N.R.A.S. 182, 97

Tuchman, Y., Sack, N., Barkat, Z. 1979. Ap. J. 234: 217

Tuchman, Y., Barkat, Z. 1980. Ap. J. 242: 199

Weaver, T.A., Zimmerman, G.B., Woosley, S.E. 1978. Ap. J. 225: 1021

Wood, P.R. 1974. Ap. J. 190: 609

Wood, P.R., Faulkner, D.J. 1986. Ap. J. 307: 659 